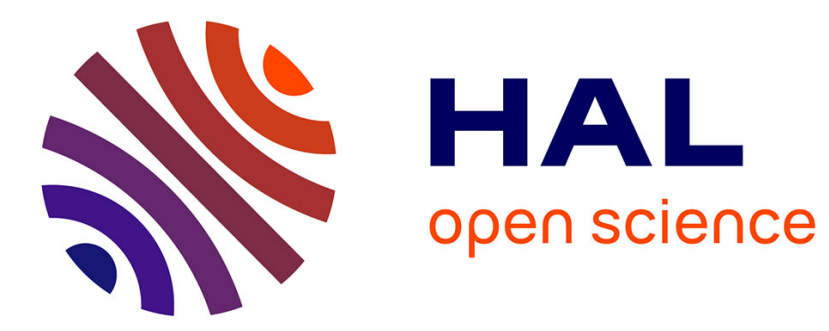

\title{
Analytical Model for the Magnetic Field Distribution in a Flux Modulation Superconducting Machine
} Alexandre Colle, T. Lubin, Sabrina Ayat, Olivier Gosselin, Jean Lévêque

\section{To cite this version:}

Alexandre Colle, T. Lubin, Sabrina Ayat, Olivier Gosselin, Jean Lévêque. Analytical Model for the Magnetic Field Distribution in a Flux Modulation Superconducting Machine. IEEE Transactions on Magnetics, 2019, 55 (12), pp.1-9. 10.1109/TMAG.2019.2935696 . hal-02376874

\section{HAL Id: hal-02376874 https://hal.science/hal-02376874}

Submitted on 22 Nov 2019

HAL is a multi-disciplinary open access archive for the deposit and dissemination of scientific research documents, whether they are published or not. The documents may come from teaching and research institutions in France or abroad, or from public or private research centers.
L'archive ouverte pluridisciplinaire HAL, est destinée au dépôt et à la diffusion de documents scientifiques de niveau recherche, publiés ou non, émanant des établissements d'enseignement et de recherche français ou étrangers, des laboratoires publics ou privés. 


\title{
Analytical Model for the Magnetic Field Distribution in a Flux Modulation Superconducting Machine
}

\author{
A. Colle, T. Lubin, S. Ayat, O. Gosselin, J. Lévêque
}

\begin{abstract}
This paper presents a theoretical analysis of an axial field machine using High Temperature Superconductors (HTS) wires and bulks. The air-gap magnetic field obtained with the HTS coil and modulated by the HTS bulks is predicted by two 2D axisymmetric models. Analytical models are based on the solution of Laplace's equation by the separation of variable method. The torque is obtained by a quick numerical integration of the Laplace force that acts on the armature winding. The proposed model is compared with 3D finite element simulations and good agreement is obtained. This model can be used with an optimization design procedure with a large reduction of the computational time.
\end{abstract}

Index Terms-Axial Field Machine, High Temperature Superconductors, Synchronous machine, Analytical Model

\section{INTRODUCTION}

$\mathrm{T}$ he electric airplanes are presented as a solution for the airtransportation of tomorrow, permitting to reduce fuel burn, noise, and emission of greenhouse gases due to the combustion of the fossil fuel [1]. The power-to-mass ratio of the electric solution should be high enough to replace the current technologies. Thus a study is being conducted here on the use of superconductors for electrical machines. In the literature, several researches were done concerning the superconducting machine in aeronautics [2]-[6]. The aim of this paper is to develop a fast analytical model to be implemented in an optimization procedure to reach the higher power-to-mass ratio.

The superconducting flux modulation machine is shown in Fig. 1. This particular topology has been studied for the radial flux topology using low temperature superconductor [7]-[8]. In this paper, we study an axial-flux machine with HTS. The HTS materials are only used for the inductor. It is composed with a solenoid coil made with HTS tape, which produces a magnetic field along the axial direction. When HTS bulks of round shape are placed in the magnetic field, electrical current is induced into the material in order to screen the magnetic field. Behind a HTS bulks, the magnetic flux is then reduced and we obtained a magnetic field modulation in the air-gap.

Manuscript received February 27, 2019. This work was supported by the Direction Générale de l'Armement (DGA).

Alexandre Colle, Thierry Lubin and Jean Lévêque are with the Group of Research in Electrical Engineering of Nancy, Faculty of Sciences and Technologies, University of Lorraine, 54506 Vandoeuvre-lès-Nancy, France (e-mail: first-name.name@univ-lorraine.fr).

Alexandre Colle is also with SAFRAN, 78114 Magny-les-Hameaux, France (e-mail: first-name.name@ safrangroup.com).

Sabrina Ayat and Olivier Gosselin are with SAFRAN, 78114 Magny-lesHameaux, France (e-mail: first-name.name@ safrangroup.com).

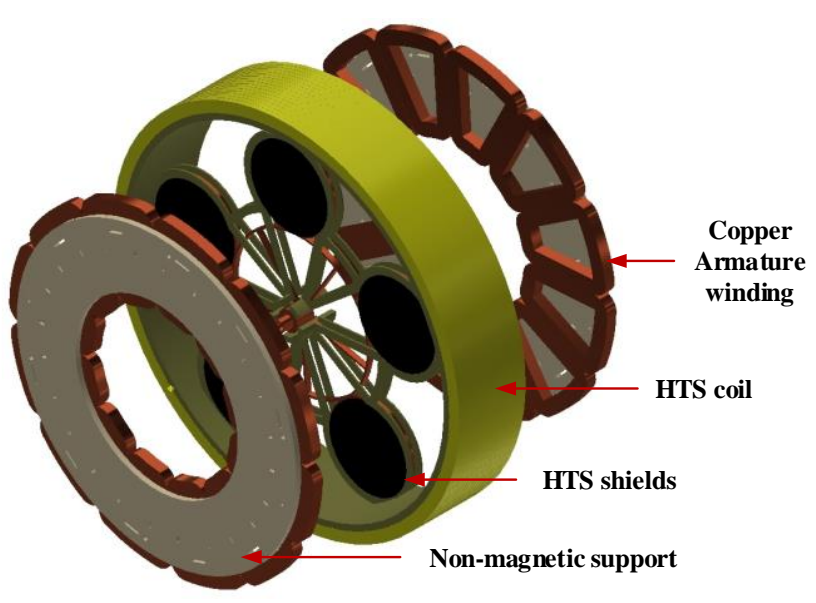

Fig. 1. Exploded view of the studied superconducting machine

Throughout the paper, the HTS bulks will be also called magnetic shield or screen.

The armature winding is made with three phase copper winding placed on each side of the bulks. The rotation of the HTS bulks gives a back-electromotive force in the stator winding due to the flux variation. No ferromagnetic material is used in this topology to decrease the total weight. So the superconducting machine is air-cored and copper coils are disposed on a non-magnetic support. In this kind of configuration, the magnetic field out of the machine is shielded actively by others superconducting coils not represented in Fig. 1. These coils are placed around the main coil. The same current in the opposite direction is driven to avoid the magnetic field of the main superconducting coil.

The magnetic field determination is a 3D problem, due to the axial structure and the flux distribution behind the screens. The screening current which expulses the magnetic flux is developed from the external surface and penetrates into the bulk. The length of penetration depends on the shield cooling, the applied magnetic field, and the critical current density of the material. The shielding effect can be calculated by:

1. Analytical techniques using the critical state model developed by Bean [9]. This method is easy to implement and fast but is limited to specific geometries with homogeneous or simple form for the critical current dependence [10].

2. Numerical methods as finite elements using a variety of formulation [11]-[16]. These methods can deal with complex geometries of superconductors but need more computational time than analytical methods. 
Due to the complexity of the studied machine, one way to compute the magnetic field is the use of numerical methods. Obviously, these methods describe accurately the flux modulation. However, the computational time needed to solve the problem is not suitable to be used in an optimization design procedure. Therefore, we propose an analytical model [17]-[20], based on some assumptions, describing the magnetic behavior for one pole pair of the superconducting inductor. The model will be accurate if the penetrations length of the screening current is small, which corresponds to the best operating point for the superconducting machine.

The paper is organized as follows. In section II, the electromagnetic problem to be solved and the assumptions are given. In section III, equations of the analytical models are solved with the separation of variable method. In section IV, the electromagnetic torque is obtained using the Laplace force. Finally, the model is validated comparing the results obtained with the analytical model with those of the FE simulations. The model is then used to study the impact of the design parameters on the machine performance, such as the air-gap length or the copper winding dimensions.

\section{PROBLEM DESCRIPTION}

As shown in Fig. 1, the axial flux superconducting machine is a $3 \mathrm{D}$ problem in an electromagnetic point of view. The natural way to solve this problem is to use a cylindrical coordinate system $(r, \theta, z)$. However, 3D analytical solutions written in cylindrical coordinate and obtained with the separation of variable method are complex and lead to numerical integration, which increases the computation time. Moreover, the bulk should have a tile shape (Fig. 2) to solve this 3D problem analytically, as was done for permanent magnets in [21]-[22]. In fact, the design of tile shape for HTS bulk with single or multi-seeds growth are restricted to small size and are usually obtained by sculpting a square or a round material. On the other hand, multi-domain round shape of HTS bulks can be bigger than $80 \mathrm{~mm}$ for the diameter [30]. This is the reason why the round shape is used for the studied machine.

To take into account the round shape for the bulks, the magnetic field distribution in the air-gap will be obtained by solving two 2D axisymmetric problems. The first analytical problem is to determine a flux modulation function which represents the shielding effect of one superconducting bulks placed in a homogeneous magnetic field. This function was already developed for the radial flux machine, but this model is not adapted for the axial flux machine [23]. A perfect diamagnetic behavior for the bulk will be considered, so no magnetic flux density penetrates into the shield. The second analytical problem is to determine the magnetic field generated by the HTS coil alone. It is then multiplied by the modulation function in order to obtain the behavior of the superconducting inductor. As shown in Fig. 3, each 2D axisymmetric problem has its own center, 0 for the HTS coil problem and 0 ' for the HTS bulk one.

Fig. 3 gives the geometrical parameters. The problem is

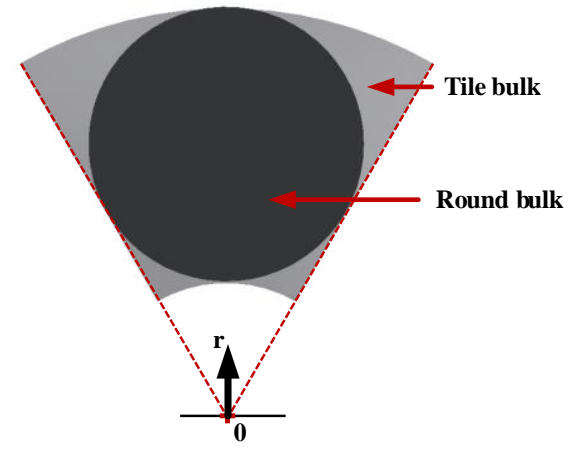

Fig. 2. Superconducting bulk with round or tile shape

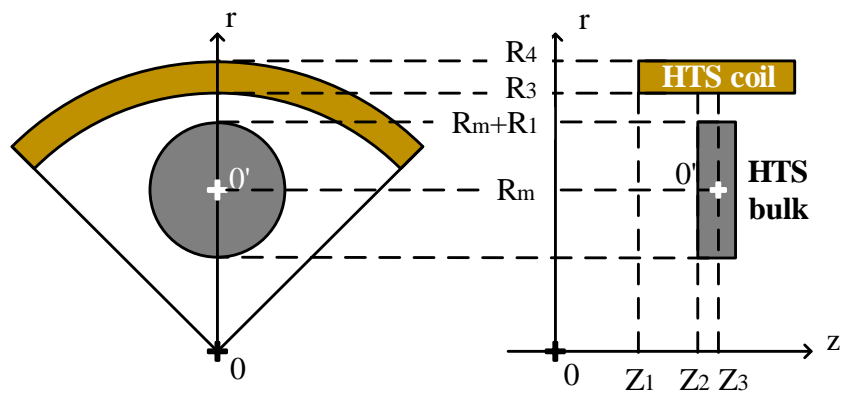

Fig. 3. Flux modulation superconducting inductor with its geometrical parameters

symmetrical around $Z_{3}$, so only half of the problem has to be solved. The inner and outer radius of the HTS coil is respectively $R_{3}$ and $R_{4}$. The radius of the HTS bulk is $R_{l}$ and its center is placed at $r=R_{m}$. The radius $R_{5}$ (not represented in Fig. 3) represents the boundary limit for the analytical modeling and is placed far from the HTS coil.

\section{SUPERCONDUCTING INDUCTOR}

\section{A. Flux modulation function}

The flux modulation function determines the behavior of the magnetic flux density around the shield. This function is obtained by the ratio of the axial component of the flux density with and without shields. The cross-section and regions of the $2 \mathrm{D}$ problem which has to be solved in order to obtain the flux modulation function is represented in Fig. 4. A permanent magnet (PM) of radius $R_{2}$ and thickness $Z_{0}$ (region I) is located far from the HTS bulk and placed on a ferromagnetic material with an infinite relative permeability. The magnet generates an axial magnetic field, which symbolize the behavior of the HTS coil added later. A roundshape superconducting bulk of radius $R_{I}$ is placed in front of the magnet. As said previously, the problem is symmetric around $Z_{3}$, so the thickness of the bulk is two times $\left(Z_{3}-Z_{2}\right)$. The radius $R_{2}$ corresponds to the external boundary for this problem. The error introduced by this artificial boundary will be analyzed later.

\section{1) Governing equations}

As shown in Fig .4, the problem is divided into three regions I, II and III. The magnetic field in each region satisfies the basic equations of magnetostatic. For the region I 


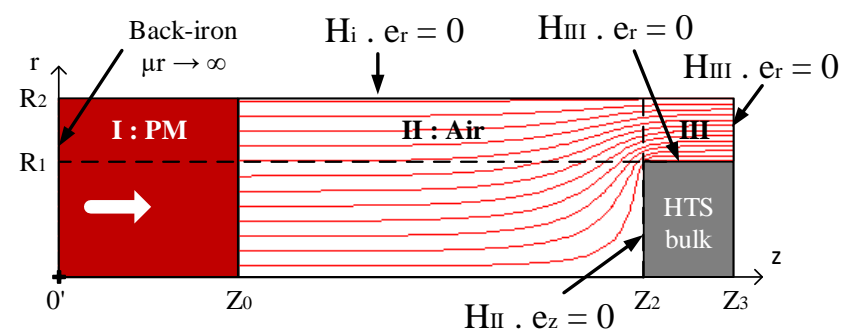

Fig. 4. Sectional view of the flux modulation function problem in the $(\mathrm{r}, \mathrm{z})$ plane, its boundary conditions and flux line with HTS bulks

(permanent magnet), a magnetic charge model is adopted. As the perfect diamagnetic behavior is considered for the HTS bulk, all the magnetic flux is deviated in the air region, numbered III. In Fig.1, the region III corresponds to the space between two HTS shields.

The direction of the PM's magnetization is represented by the white arrow in Fig. 4. This is a volume magnetization but it can be replaced by an equivalent surface magnetic charge density at $z=Z_{0}$

$$
\sigma_{\text {magnet }}=B_{r} / \mu_{0}
$$

where $B_{r}$ is the axial remanent induction of the magnet.

For this problem, the Maxwell equations are as follow

$$
\nabla \cdot \overrightarrow{\mathbf{B}_{\mathbf{i}}}=0 \quad \vec{\nabla} \times \overrightarrow{\mathbf{H}_{\mathbf{i}}}=0 \quad(i=I, \text { II or III })
$$

where $B_{i}$ and $H_{i}$ are the magnetic flux density and the magnetic field in region $i$. The magnetic field can be written in term of a magnetic scalar potential $\Phi_{i}$, since no current distribution is involved.

$$
\overrightarrow{\mathbf{H}_{\mathbf{i}}}=-\vec{\nabla} \Phi_{i} \quad(i=I, I I \text { or } I I I)
$$

Equations (2) and (3) are combined to give the following Laplace equation written in the 2D cylindrical coordinates.

$$
\frac{\partial \Phi_{i}^{2}}{\partial r^{2}}+\frac{1}{r} \frac{\partial \Phi_{i}}{\partial r}+\frac{\partial \Phi_{i}^{2}}{\partial z^{2}}=0
$$

\section{2) Boundary conditions}

Some boundary conditions have to be defined in the $r$ - and $z$-directions to solve this problem. The diamagnetic behavior of bulks acts as a flux barrier which imposed the normal component of the flux density equal to zero at its boundaries:

$$
\begin{gathered}
B_{z}\left(0<r<R_{1}, Z_{2}\right)=0 \\
B_{r}\left(R_{1}, Z_{2}<z<Z_{3}\right)=0
\end{gathered}
$$

where $B_{z}$ and $B_{r}$ represents respectively the axial and radial component of the flux density. Moreover, in order to obtain a purely axial direction for the magnetic flux produced by the magnet alone, the normal component of the magnetic field is imposed equal to zero at all others boundaries and is finite at $r=0$.

$$
\begin{gathered}
H(0, z) \text { is finite } \\
H_{r}\left(R_{2}, z\right)=0 \\
H_{r}(r, 0)=0 \\
H_{r}\left(R_{1}<r<R_{2}, Z_{3}\right)=0
\end{gathered}
$$

In each region, the relative permeability is equal to 1 . So from (5) to (10), the boundary condition can be re-written in terms of the magnetic scalar potential in order to find the eigenvalue of the problem defined in the $r$-direction.

$$
\left\{\begin{array}{l}
\left.\frac{\partial \Phi}{\partial r}\right|_{r=R_{1}}=0 \quad \mathrm{Z}_{2}<\mathrm{z}<\mathrm{Z}_{3} \\
\Phi(0, z) \text { is finite } \\
\left.\frac{\partial \Phi}{\partial r}\right|_{r=R_{2}}=0
\end{array}\right.
$$

\section{3) General solution in each region}

By using the separation of variable method, a general solution of (4) is obtained by satisfying the boundary conditions (11). The general solution is the same for regions I and II and can be written as

$$
\begin{gathered}
\Phi_{i}(r, z)=A_{i 0} z+B_{i 0} \\
+\sum_{k=1}^{\infty}\left[A_{i k} e^{\beta_{k} z}+B_{i k} e^{-\beta_{k} z}\right] J_{0}\left(\beta_{k} r\right) \\
(i=I \text { or } I I)
\end{gathered}
$$

with

$$
\beta_{k}=\frac{X_{k}}{R_{2}}
$$

where $X_{k}$ is the $k$ th zero of the derivative of the Bessel function $J_{0}\left(X_{k}\right)$. Coefficients $A_{i 0}, B_{i 0}, A_{i k}$ and $B_{i k}$ will be determined using the interface conditions.

The general solution for the modulate region III is slightly different, the boundary conditions (11) at $r=R_{l}$ and $r=R_{2}$ are used to obtain the eigenvalues. It can be written as

$$
\begin{gathered}
\Phi_{I I I}(r, z)=A_{I I I 0} z+B_{I I I 0} \\
+\sum_{l=1}^{\infty}\left[A_{I I I, l} e^{\alpha_{l} z}+B_{I I I, l} e^{-\alpha_{l} z}\right] \\
\times\left(J_{0}\left(\alpha_{l} r\right) Y_{1}\left(\alpha_{l} R_{1}\right)-J_{1}\left(\alpha_{l} R_{1}\right) Y_{0}\left(\alpha_{l} r\right)\right)
\end{gathered}
$$

where $\alpha_{l}$ is the lth zero of the cross product of the Bessel function.

$$
J_{1}\left(\alpha_{l} R_{1}\right) Y_{1}\left(\alpha_{l} R_{2}\right)-J_{1}\left(\alpha_{l} R_{2}\right) Y_{1}\left(\alpha_{l} R_{1}\right)=0
$$

The unknown coefficients $A_{I I I 0}, B_{I I I 0}, A_{I I I l}$ and $B_{I I I l}$ will be determined using the interface conditions. 


\section{4) Unknown coefficient determination}

Equations (12) and (14) show that the problem presents six unknown coefficients per harmonic. To solve this problem, we need six independents linear equations. Two equations are obtained by the remaining boundary conditions (9) and (10). The tangential component of the magnetic field strength is continuous through a region interface. The normal component at $z=Z_{0}$ is not continuous due to the surface magnetic charge of the magnet. The normal component at $z=Z_{2}$ is given by (5) between $r=0$ and $r=R_{l}$ and is continuous elsewhere.

$$
\begin{array}{cc}
\text { at } z=0: & \Phi_{I}=0 \\
\text { at } z=Z_{0}: & \frac{\partial \Phi_{I}}{\partial z}-\frac{\partial \Phi_{I I}}{\partial z}=\sigma_{\text {magnet }} \\
& \Phi_{I}=\Phi_{I I} \\
\text { at } z=Z_{2}: & \frac{\partial \Phi_{I I}}{\partial z}=\left\{\begin{array}{c}
\left.\frac{\partial \Phi_{I I I}}{\partial z}\right|_{R_{1}<r<R_{2}} \\
0 \text { elsewhere }
\end{array}\right. \\
& \left.\Phi_{I I}\right|_{R_{1}<r<R_{2}}=\left.\Phi_{I I I}\right|_{R_{1}<r<R_{2}}
\end{array}
$$$$
\text { at } z=Z_{3} \text { : } \quad \Phi_{I I I}=0
$$

Using these six linear equations, all unknown constants can be obtained. Developments are given in the Appendix A. It is worth noting that the zero order Bessel function integrations involved during the coefficients computation has an analytical form [24].

\section{5) Definition of the flux modulation function}

Once the magnetic scalar potential is known in each region, the axial and the radial component of the flux density can be computed in the air-gap region (II).

$$
\begin{aligned}
& B_{I I}(r, z)=-\mu_{0} \frac{\partial \Phi_{I I}}{\partial z} \\
& B_{I I r}(r, z)=-\mu_{0} \frac{\partial \Phi_{I I}}{\partial r}
\end{aligned}
$$

The flux modulation function is the ratio of (17) and the value of the magnetic flux density without shield. This value can be simply obtained by using the Ampere law.

$$
B_{0}=\frac{\mu_{0} \sigma_{P M}}{\left(1+\frac{\left(Z_{3}-Z_{0}\right)}{Z_{0}}\right)}
$$

Finally, the flux modulation function is given by

$$
F M(r, z)=\frac{B_{I I z}(r, z)}{B_{0}}
$$

\section{B. HTS coil problem}

The cross-section of the HTS coil problem and the different regions are given in Fig. 5. To simplify the analysis, the coordinates system is translated axially by $z=-Z_{3}$ compare to

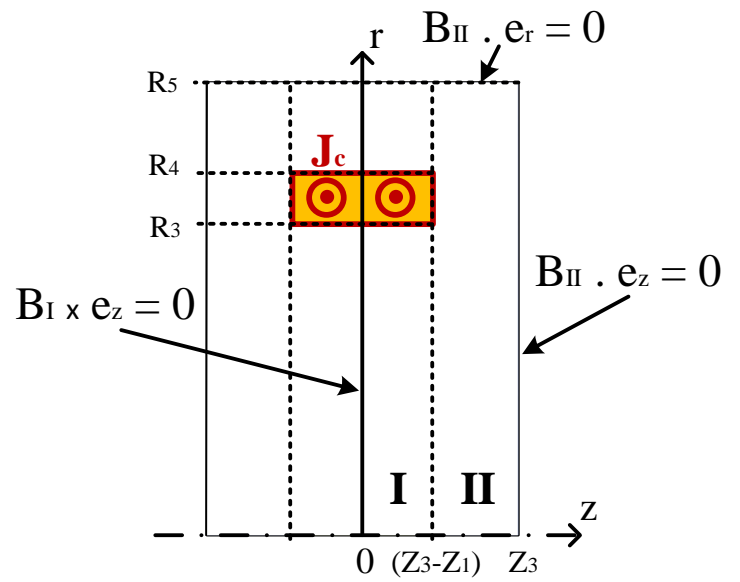

Fig. 5. Sectional view of the superconducting coil problem in the $(\mathrm{r}, \mathrm{z})$ plane and its boundary conditions

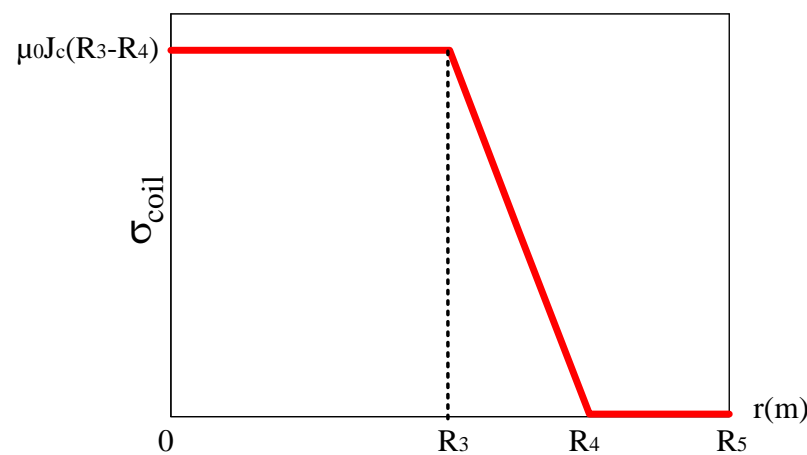

Fig. 6. Equivalent surface magnetic charge distribution at $z=Z_{3}-Z_{l}$ along the $r$-direction

Fig. 3. The symmetry of the problem is used to reduce the number of regions. For region I, the current density is applied between $r=R_{2}$ and $r=R_{3}$ and between $z=0$ and $z=\left(Z_{3}-Z_{1}\right)$. The region II corresponds to the air domain with no current.

\section{1) Governing equations}

A Coulombian approach is used to describe the current distribution [25]. This method allows us to work directly with a magnetic scalar potential formulation. Therefore, the current density in the coil is replaced by an equivalent surface magnetic charge density $\sigma_{\text {coil }}$ at $z=Z_{3}-Z_{1}$, as shown in Fig. 6 .

$$
\sigma_{\text {coil }}(r)=\left\{\begin{array}{cl}
\mu_{0} J_{c}\left(R_{4}-R_{3}\right) & 0<r<R_{3} \\
\mu_{0} J_{c}\left(R_{4}-r\right) & \mathrm{R}_{3}<r<R_{4} \\
0 & r>R_{4}
\end{array}\right.
$$

where $J_{c}$ is the critical current density in the HTS coil.

The surface magnetic charge density drawn in Fig. 6 can be developed into Bessel-Fourier series as follows

$$
\sigma_{\text {coil }}(r)=\sum_{m=1}^{\infty} \sigma_{c m} J_{0}\left(\gamma_{m} r\right)
$$

with

$$
\begin{gathered}
\sigma_{c m}=\frac{2}{R_{5}^{2}\left(J_{0}\left(\gamma_{m} R_{5}\right)\right)^{2}}\left(\int_{0}^{R_{3}} \mu_{0} J_{c}\left(R_{4}-R_{3}\right) r J_{0}\left(\gamma_{m} r\right) d r\right. \\
\left.\quad+\int_{R_{3}}^{R_{4}} \mu_{0} J_{c}\left(R_{4}-r\right) r J_{0}\left(\gamma_{m} r\right) d r\right)
\end{gathered}
$$


The analytical forms of the integrals in (23) are given in appendix B. For region I and II, the Laplace equation has to be solved.

$$
\frac{\partial \Phi_{i}^{2}}{\partial r^{2}}+\frac{1}{r} \frac{\partial \Phi_{i}}{\partial r}+\frac{\partial \Phi_{i}^{2}}{\partial z^{2}}=0
$$

\section{2) Boundary conditions}

As shown in Fig. 5, boundaries are placed such as $R_{5}>>R_{4}$ and $Z_{3}>>\left(Z_{3}-Z_{1}\right)$ and act as infinite boundary conditions. This imposes the normal component of the magnetic field to be zero

$$
\begin{gathered}
H_{z}\left(0<r<R_{5}, Z_{3}\right)=0 \\
H_{r}\left(0<r<R_{5}, 0\right)=0 \\
H_{r}\left(R_{5}, 0<z<Z_{3}\right)=0
\end{gathered}
$$

Moreover, the magnetic field has to be finite at $r=0$.

$$
H(0, z) \text { is finite }
$$

From (27) and (28), the boundary conditions can be rewritten in terms of the magnetic scalar potential in order to find the eigenvalue of the problem in the $r$-direction.

$$
\left\{\begin{array}{c}
\left.\frac{\partial \Phi}{\partial r}\right|_{r=R_{5}}=0 \\
\Phi(0, z) \text { is finite }
\end{array}\right.
$$

\section{3) General solution in the each region}

A general solution of (24) is obtained satisfying the boundary conditions (29) by using the method of separation of variables. The general solution is common for regions I and II and can be written as

$$
\begin{gathered}
\Phi_{i}(r, z)=C_{i 0} z+D_{i 0} \\
+\sum_{m=1}^{\infty}\left[C_{i m} e^{\gamma_{m} z}+D_{i m} e^{-\gamma_{m} z}\right] J_{0}\left(\gamma_{m} r\right) \\
(i=I \text { or } I I)
\end{gathered}
$$

with

$$
\gamma_{m}=\frac{X_{m}}{R_{5}}
$$

where $X_{m}$ is $m$ th zero of the derivative of the Bessel function $J_{0}\left(X_{m}\right)$. Unknown coefficients $C_{i 0}, D_{i 0}, C_{i m}$ and $D_{i m}$ will be determined with interface conditions.

\section{4) Unknown coefficient determination}

Equations (30) show that the problem has four unknown coefficients per harmonic. Two equations are obtained by the remaining boundary conditions (25) and (26). The two others equations are given by the interface conditions at $z=\left(Z_{3}-Z_{1}\right)$. It corresponds to the continuity of the tangential component of the magnetic field and the continuity of the normal component of the flux density a)

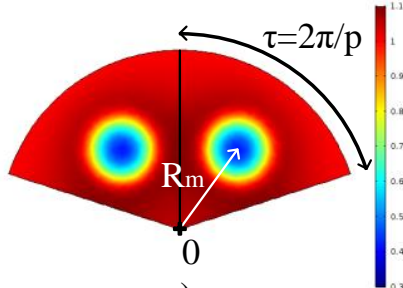

b)

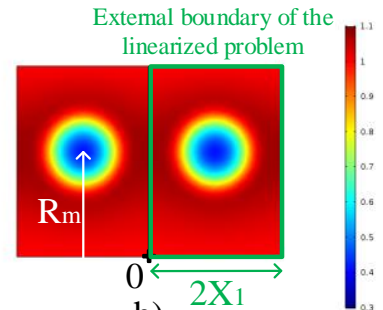

Fig. 7. Magnetic flux density shielded by HTS bulks for (a) cylindrical problem (b) linearized problem

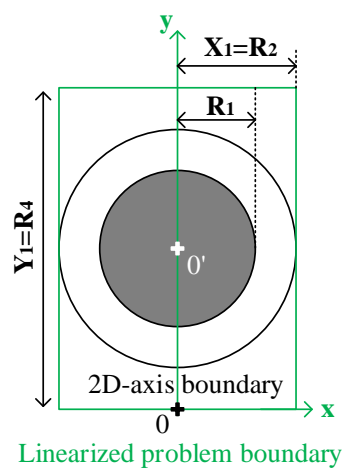

Fig. 8. 2D-axis external boundary at $r=R_{2}$ compared to the linearized problem external boundary

$$
\begin{array}{rlrl}
\text { at } z=0: & \Phi_{I}=0 \\
\text { at } z=Z_{3}-Z_{I}: & \frac{\partial \Phi_{I}}{\partial z}-\frac{\partial \Phi_{I I}}{\partial z} & =\sigma_{\text {coil }}(r) \\
& & \Phi_{I}=\Phi_{I I} \\
\text { at } z=Z_{3}: & \frac{\partial \Phi_{I I}}{\partial z}=0
\end{array}
$$

Using these four linear equations, all unknown constants can be obtained. Developments are given in appendix C.

5) Axial magnetic field from the scalar potential

Once the scalar potential is known in each region, the axial component of the flux density can be obtained in region I, where the stator windings will be placed.

$$
B_{\text {coilz }}(r, z)=-\mu_{0} \frac{\partial \Phi_{I}}{\partial z}+\sigma_{\text {coil }}(r)
$$

\section{Global solution: field of the HTS inductor}

In order to deal with a simpler expression of the Laplace force, the cylindrical topology is linearized. In Fig. 7, the magnetic flux density behind an HTS shield is shown for a cylindrical and a linearized problem around the mean radius $R_{m}$. Both configurations give the same flux modulation value, so the curvature effects can be neglected without an important error. The r-coordinate of the $2 \mathrm{D}$-axisymmetric problems is then transformed into the $\mathrm{x}$ - and $\mathrm{y}$-coordinate by

$$
r^{2}=x^{2}+y^{2}
$$

The center of both problem is not the same, so a translation of the mean radius is done in the flux modulation function. 
Finally, the magnetic behavior of the superconducting inductor is given by

$$
B_{z}(x, y, z)=F M\left(r-R_{m}, z\right) \times B_{\text {coil }}\left(r, z-Z_{3}\right)
$$

where $r$ is given by (34) and the function FM by (20).

The expression of the tangential component can be obtained as follow

$$
B_{\Theta}(x, y, z)=F M_{2}\left(r-R_{m}, z\right) \times B_{\text {coilz }}\left(r, z-Z_{3}\right)
$$

with

$$
F M_{2}(r, z)=\frac{B_{I I r}(r, z) \cos (\theta)}{B_{0}}
$$

Moreover $\theta=\tan ^{-1}(y / x), \quad r$ is still given by (34) and $B_{0}$ is given by (19). This tangential component of the magnetic field has no influence on the electromagnetic torque. It is however required to account for some important computation such as the eddy-current losses in the stator coils [26].

As explained previously and shown in Fig. 8, the external boundary of the modulation function problem $R_{2}$ is different from the boundary of the linearized problem $\left(X_{1}, Y_{l}\right)$. So for every point located at $r>R_{2}$, the value of the modulation function (20) at $r=R_{2}$ is imposed. The error introduced by the $2 \mathrm{D}$-axisymmetric boundary is investigated in the next part.

\section{EleCtRomagnetic TORQUE CALCULATION}

The electromagnetic torque is obtained using the $\mathrm{x}$ component of the Laplace force from the center of the machine. In the case of an axial flux machine, the radial current density of the armature winding and the axial magnetic flux density of the inductor generate this stress. For the linearized axial machine, the radial direction of the current density is transformed in the y-coordinate. The force is then numerically integrated over the copper coils.

$$
T_{e}=\iiint_{V} y B_{z} J_{y} d V
$$

The y component of the current density in (38) is obtained by a 2 -D Fourier series, defined as

$$
J_{y}(x, y)=\sum_{m=1}^{\infty} \sum_{n=1}^{\infty} J_{m n y} \cos \left(w_{n} x\right) \cos \left(w_{m} y\right)
$$

where

$$
\begin{aligned}
& w_{n}=\frac{2 n \pi}{D_{x}} \quad n=1,3,5, \ldots \\
& w_{m}=\frac{m \pi}{2 R_{5}} \quad \mathrm{~m}=1,3,5, \ldots
\end{aligned}
$$

The coefficient $J_{m n y}$ depends on the type of armature winding used (distributed or concentric), as exposed in [27].

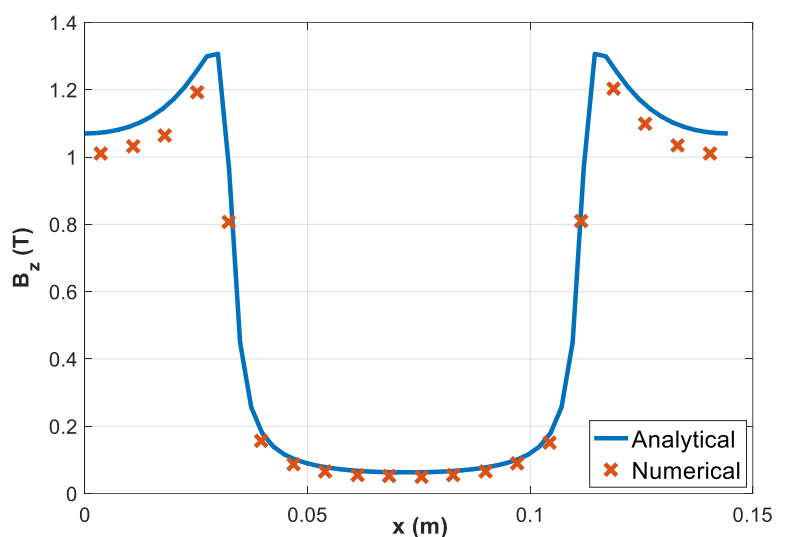

Fig. 9. Axial component of the flux density along the $x$-coordinate $5 \mathrm{~mm}$ behind the screen

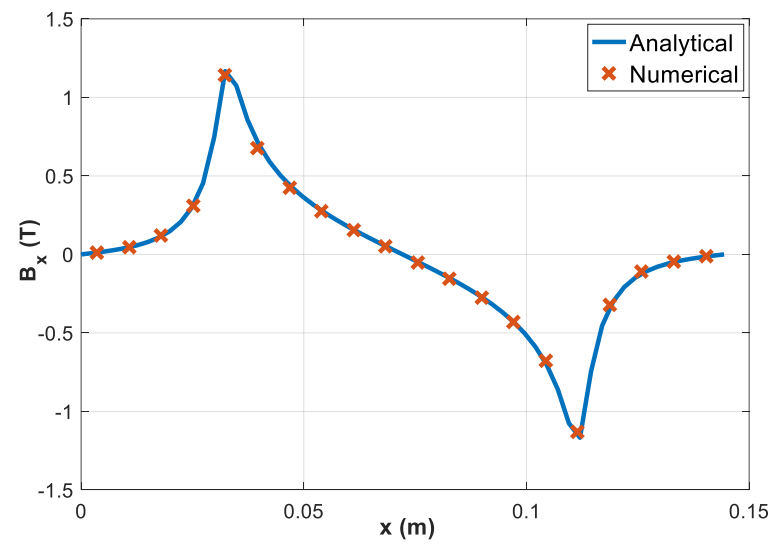

Fig. 10. Tangential component of the flux density along the $x$-coordinate $5 \mathrm{~mm}$ behind the screen

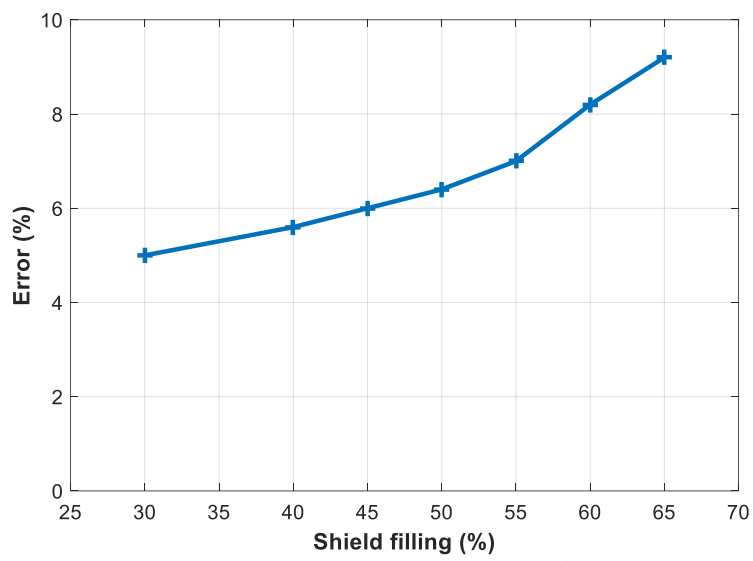

Fig. 11. Maximal error on $\mathrm{B}_{z}$ versus the HTS bulk filling at $y=R_{m}$

The axial component of the magnetic flux density is then obtained from (35).

$$
\begin{gathered}
B_{z}(x, y, z)= \\
\mu_{0}\left(\frac{A_{I 0}-\sigma_{P M}+\sum_{k=1}^{\infty} A_{I k} \beta_{k} \operatorname{ch}\left(\beta_{k} z\right) J_{0}\left(\beta_{k} r\right)}{B_{0}}\right) \\
\times\left(J_{c}\left(R_{4}-R_{3}\right)+\sum_{m=1}^{\infty} K_{I I \mathrm{~m}} \gamma_{m} \operatorname{ch}\left(\gamma_{m} z\right) J_{0}\left(\gamma_{m} r\right)\right)
\end{gathered}
$$


where $\sigma_{P M}$ is the equivalent surface magnetic charge density of the magnet in the modulation function problem, $B_{0}$ is given by (19) and $J_{c}$ is the critical current density of the HTS coil. The constant $K_{I I m}$ is given by

$$
K_{I I \mathrm{~m}}=\frac{\sigma_{c m}}{\gamma_{m} \operatorname{sh}\left(\gamma_{m} Z_{1}\right)} \frac{\operatorname{th}\left(\gamma_{m}\left(Z_{1}-Z_{2}\right)\right) \times \operatorname{th}\left(\gamma_{m} Z_{1}\right)}{\operatorname{th}\left(\gamma_{m}\left(Z_{1}-Z_{2}\right)\right)-\operatorname{th}\left(\gamma_{m} Z_{1}\right)}
$$

where $\sigma_{c m}$ is the equivalent surface magnetic charge density of the HTS coil problem.

TABLE I

DESIGN PARAMETERS OF THE SUPERCONDUCTING MACHINE

\begin{tabular}{ccc}
\hline Type & Symbol & Value \\
\hline Poles-pairs & $\mathrm{p}$ & 5 \\
HTS coil diameter & $2 \mathrm{R}_{4}$ & $353 \mathrm{~mm}$ \\
HTS coil length & $2\left(\mathrm{Z}_{3}-\mathrm{Z}_{1}\right)$ & $69 \mathrm{~mm}$ \\
HTS coil thickness & $\mathrm{R}_{4}-\mathrm{R}_{3}$ & $10,5 \mathrm{~mm}$ \\
HTS bulk radius & $\mathrm{R}_{1}$ & $40 \mathrm{~mm}$ \\
HTS bulk center & $\mathrm{R}_{\mathrm{m}}$ & $114,5 \mathrm{~mm}$ \\
Total air-gap & $\mathrm{g}$ & $4,5 \mathrm{~mm}$ \\
Critical current density & $\mathrm{J}_{\mathrm{c}}$ & $230 \mathrm{~A} / \mathrm{mm}$ \\
Outer radius copper coil & $\mathrm{R}_{\mathrm{o}}$ & $145 \mathrm{~mm}$ \\
Inner radius copper coil & $\mathrm{R}_{\mathrm{i}}$ & $84 \mathrm{~mm}$ \\
Thickness copper coil & $\mathrm{E}$ & $9,5 \mathrm{~mm}$ \\
Width copper coil & $\mathrm{W}$ & $13 \mathrm{~mm}$
\end{tabular}

\section{RESULTS OBTAINED WITH THE ANALYTICAL MODEL}

In this section, the air-gap magnetic field distribution and the electromagnetic torque obtained with the proposed analytical model is compared with 3D FE simulations (COMSOL Multiphysics@) considering the actual geometry of the studied machine (Fig. 1). For each position of the rotor, the electromagnetic torque is calculated using (38). Then, the influence of some geometrical parameters like the air-gap length or the ratio between inner and outer radius of the armature winding is analyzed and compared with typical values. The geometrical parameters of the studied machine are given in the Table I.

\section{A. Magnetic field distribution}

Figs. 9 and 10 respectively show the axial and tangential component of the flux density at $r=R_{m}$ and $z=Z_{2}-g / 2$, where $g$ is the air-gap length. The shielding effect of the HTS bulks is well observed in Fig. 9, the magnetic flux density is reduced by more than $90 \%$ behind the screen.

The 3D HTS inductor using Table I parameters, is also solved using FE software with cylindrical bulks. As shown in Figs. 9 and 10, a good matching is found between numerical and analytical results. The HTS bulk radius $R_{l}$ is fixed to 40 $\mathrm{mm}$ and the value of $R_{2}$ is $72 \mathrm{~mm}$. The shield filling is defined by the ratio $R_{1} / R_{2}$. For our problem, its value is equal to $55 \%$. With this shield filling value, the maximal error between the analytical model and the 3D FE simulation is equal to $7 \%$, as shown in Fig. 11.

Fig. 11 shows that the maximal error grows with the shield filling. This result is predictable because the magnetic flux density is fixed by the external boundaries. When $R_{1} \approx R_{2}$

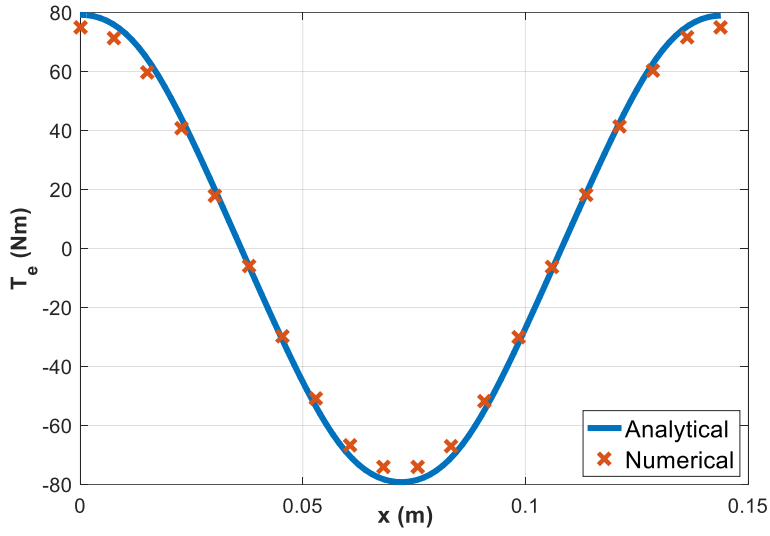

Fig. 12. Analytical and numerical results for the electromagnetic torque

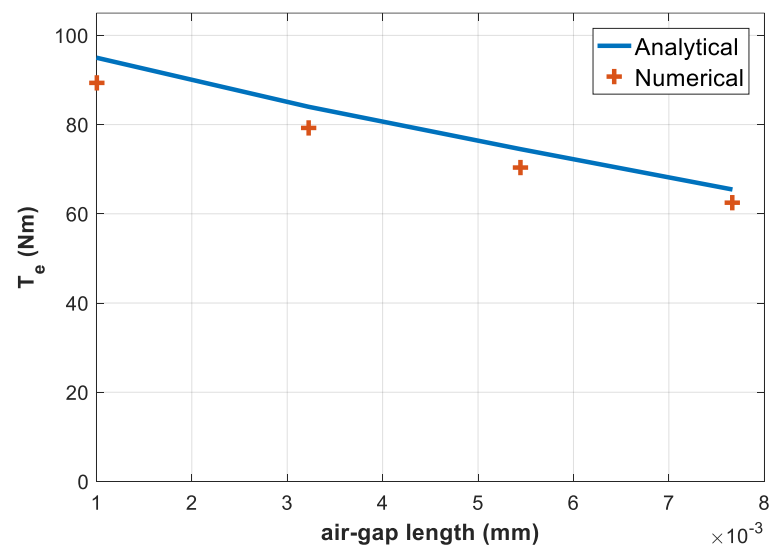

Fig. 13. Variation of the electromagnetic torque versus the air-gap length

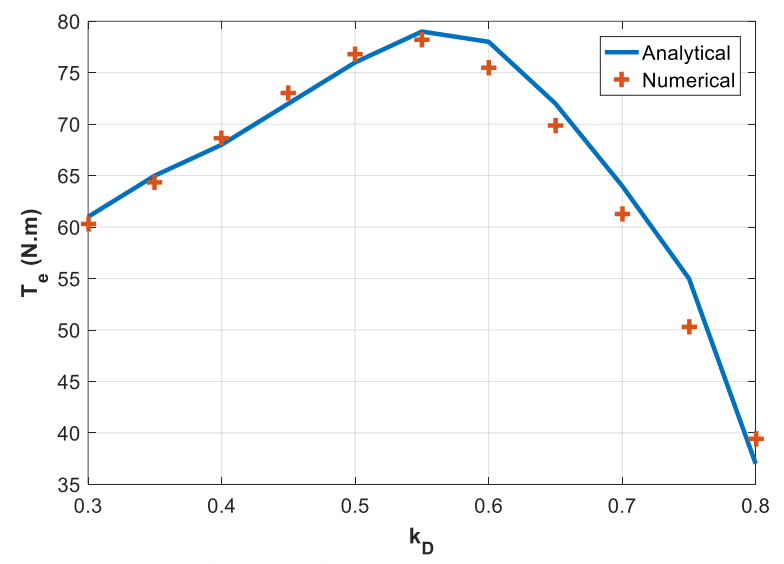

Fig. 14. Torque as a function of the ratio $R_{i} / R_{o}$

(shield filling $\approx 100 \%$ ), the flux passes through the thin band between these radii which increase the value of the magnetic field. However, with the linearized external boundary the flux has the opportunity to pass above and below the bulk and the magnetic field doesn't increase in the same way. That's why the value of the axial magnetic flux density is higher with the analytical model (Fig. 9). The difference between the 2Daxisymmetric external boundary of the flux modulation problem and the linearized problem is acceptable until a shield filling of $65 \%$. Hopefully, the electromagnetic torque is higher 
for this kind of machine where the shield filling is around $50 \%$, which corresponds to a region where the analytical model is accurate.

\section{B. Electromagnetic torque}

The electromagnetic torque is calculated over a periodicity length and is shown in Fig. 12. The error between the analytical model and numerical simulations is lower than $4 \%$. The full load condition is obtained at $x=0$. For this position, the torque reaches $79 \mathrm{Nm}$ using the analytical model and 76 Nm with the 3D FE simulations.

By using the Laplace force calculation, the reluctance torque of the machine is neglected. This is not a problem for the studied machine because the reluctance torque is very low due to its large air-gap. However, this should be considered in a machine with a thinner air-gap [28].

The length of the air-gap has a significant importance on the performance of a superconducting machine. Fig. 13 shows the torque as a function of the air-gap length. It results that the torque decreases quickly with this length, so special care should be taken in reducing its value during the design. The power of the superconducting machine decreases by $20 \%$ when the air-gap increase from $1 \mathrm{~mm}$ (conventional machine) to $4,5 \mathrm{~mm}$ (partial superconducting machine) because of the thickness of the thermal insulation (cryostat and vacuum). This loss is compensated by the high magnetic flux density obtained with the HTS wire. Fig. 13 shows that the proposed analytical model remains accurate for large value of the airgap.

The electromagnetic torque is then plotted as a function of the armature winding radius ratio $k_{D}=R_{i} / R_{o}$. Fig. 14 shows that the torque has a maximum value for $k_{D}=55 \%$. This behavior is classical for an axial flux machine and can be found analytically [29] with a simple formula (44) which gives the maximum torque value for a radius ratio $k_{D}=57.7 \%$.

$$
T=2 \pi B_{\mathrm{m}} A_{i} R_{o}^{3} k_{D}\left(1-k_{D}{ }^{2}\right)
$$

where $B_{m}$ is the maximum value of the air-gap flux density, $A_{i}$ is the electrical load at the mean radius, $R_{o}$ is the outer radius of the armature winding.

Once again, we can observe in Fig. 14 that good agreement is obtained between the analytical model and the $3 \mathrm{D}$ FE simulations. The analytical model is much faster than the numerical one. It takes only $0,5 \mathrm{~s}$ with the proposed model for ten points of calculation whereas $72 \mathrm{~s}$ is needed for the finite element method.

\section{CONCLUSION}

In this paper, an analytical model for computing the magnetic behavior of a flux modulation superconducting machine is developed. The magnetic field distribution is obtained with two 2D axisymmetric analytical models.

Even if the real problem is purely $3-\mathrm{D}$, this approach gives accurate results. We have seen that the maximal error on the electromagnetic torque is less than $4 \%$ for a given geometry.
Another important result concerns the computational time. The torque computation obtained with the analytical is 140 times faster than the 3-D FE simulation. This analytical model is a useful tool to be included in an optimization procedure.

\section{APPENDIX}

A-Unknown coefficients determination:

From (16), we obtain a system of six equations.

-for the mean value term:

at $z=0: \quad B_{I 0}=0$

at $z=Z_{0}: \quad A_{I 0}-A_{I I 0}=\sigma_{\text {magnet }}$

$$
\begin{array}{ll} 
& A_{I 0} Z_{1}+B_{I 0}=A_{I I 0} Z_{1}+B_{I 0 I} \\
\text { at } z=Z_{2}: & A_{I I 0} R_{2}^{2}=A_{I I I 0}\left(R_{2}^{2}-R_{1}^{2}\right) \\
& A_{I I I 0} Z_{2}+B_{I I I 0}=A_{I I 0} Z_{2}+B_{I I 0} \\
\text { at } z=Z_{3}: & A_{I I I 0} Z_{3}+B_{I I I 0}=0
\end{array}
$$

-for harmonic terms:

$$
\begin{array}{ll}
\text { at } z=0: & A_{I k}+B_{I k}=0 \\
\text { at } z=Z_{0}: & A_{I k} e^{\beta_{k} Z_{0}}-B_{I k} e^{-\beta_{k} Z_{0}}=A_{I l k} e^{\beta_{k} Z_{0}}-B_{I l k} e^{-\beta_{k} Z_{0}} \\
& A_{I l} e^{\beta_{k} Z_{0}}+B_{I k} e^{-\beta_{k} Z_{0}}=A_{I l k} e^{\beta_{k} Z_{0}}+B_{I l k} e^{-\beta_{k} Z_{0}} \\
\text { at } z=Z_{2}: & \left(A_{I l k} e^{\beta_{k} Z_{2}}-B_{I l k} e^{-\beta_{k} Z_{2}}\right) K_{1}=A_{I I I 0} K_{2}+\sum_{l=1}^{\infty}\left(A_{I I I} e^{\alpha_{l} Z_{2}}-B_{I I I} e^{-\alpha_{l} Z_{2}}\right) \alpha_{l} K_{3} \\
& \left(A_{I I I l} e^{\alpha_{l} Z_{2}}+B_{I I I} e^{-\alpha_{l} Z_{2}}\right) K_{4}=\sum_{k=1}^{\infty}\left(A_{I l k} e^{\beta_{k} Z_{2}}+B_{I l k} e^{-\beta_{k} Z_{2}}\right) K_{3}
\end{array}
$$

at $z=Z_{3}: \quad A_{I I I l} e^{\alpha_{1} Z_{3}}+B_{I I I l} e^{-\alpha_{l} Z_{3}}=0$

where

$K_{1}=\beta_{k} \frac{R_{2}^{2}}{2} J_{0}^{2}\left(\beta_{k} R_{2}\right)$

$K_{2}=\frac{-R_{1}}{\beta_{k}} J_{1}\left(\beta_{k} R_{1}\right)$

$K_{3}=\left(F J\left(\alpha_{l}, \beta_{k}, R_{1}, R_{2}\right) Y_{1}\left(\alpha_{l} R_{1}\right)-F Y\left(\alpha_{l}, \beta_{k}, R_{1}, R_{2}\right) J_{1}\left(\alpha_{l} R_{1}\right)\right)$

$K_{4}=\left[\frac{r^{2}}{2}\left(J_{0}^{2}\left(\alpha_{l} r\right)+J_{1}^{2}\left(\alpha_{l} r\right)+Y_{0}^{2}\left(\alpha_{l} r\right)+Y_{1}^{2}\left(\alpha_{l} r\right)+\ldots\right.\right.$

$$
\left.\left.\ldots J_{0}\left(\alpha_{l} r\right) Y_{0}\left(\alpha_{l} r\right)+J_{1}\left(\alpha_{l} r\right) Y_{1}\left(\alpha_{l} r\right)\right)\right]_{R_{1}}^{R_{2}}
$$

$F J\left(\alpha_{l}, \beta_{k}, R_{1}, R_{2}\right)=\left[\frac{\alpha_{l} r J_{1}\left(\alpha_{l} r\right) J_{0}\left(\beta_{k} r\right)-\beta_{k} r J_{0}\left(\alpha_{l} r\right) J_{1}\left(\beta_{k} r\right)}{\alpha_{l}{ }^{2}-\beta_{k}{ }^{2}}\right]_{R_{1}}^{R_{2}}$

$F Y\left(\alpha_{l}, \beta_{k}, R_{1}, R_{2}\right)=\left[\frac{\alpha_{l} r Y_{1}\left(\alpha_{l} r\right) J_{0}\left(\beta_{k} r\right)-\beta_{k} r Y_{0}\left(\alpha_{l} r\right) J_{1}\left(\beta_{k} r\right)}{\alpha_{l}^{2}-\beta_{k}^{2}}\right]_{R_{1}}^{R_{2}}$

$\underline{B}$-Bessel function integration rules:

$\int_{0}^{Y_{2}} r J_{0}\left(\gamma_{m} r\right) d r=Y_{2} J_{1}\left(\gamma_{m} Y_{2}\right)$

$\int r^{2} J_{0}(r) d r=r^{2} J_{1}(r)-\frac{\pi r}{2}\left[J_{1}(r) H_{0}(r)-J_{0}(r) H_{1}(r)\right]$

where

$H_{v}(r)$ denotes the Struve function of order $v$. 
C-Unknown coefficient determination:

From (32), we obtain a system of four equations.

-for the mean value term:

at $z=0: \quad D_{I 0}=0$

at $z=Z_{3}-Z_{1}: \quad C_{I 0}-C_{I I 0}=0$

$C_{I 0}\left(Z_{3}-Z_{1}\right)+D_{I 0}-\left(C_{I I 0}\left(Z_{3}-Z_{1}\right)+D_{I I 0}\right)=0$

at $z=Z_{3}: \quad D_{I I 0}=0$

-for harmonic terms:

$$
\begin{array}{ll}
\text { at } z=0: & C_{\mathrm{Im}}+D_{\mathrm{Im}}=0 \\
\text { at } z=Z_{3}-Z_{I}: & C_{I \mathrm{~m}} e^{\gamma_{m}\left(Z_{3}-Z_{1}\right)}+D_{I \mathrm{~m}} e^{-\gamma_{m}\left(Z_{3}-Z_{1}\right)}=C_{I I \mathrm{~m}} e^{\gamma_{m}\left(Z_{3}-Z_{1}\right)}+D_{I I \mathrm{~m}} e^{-\gamma_{m}\left(Z_{3}-Z_{1}\right)} \\
& C_{I I \mathrm{~m}} e^{\gamma_{m}\left(Z_{3}-Z_{1}\right)}-D_{I I \mathrm{~m}} e^{-\gamma_{m}\left(Z_{3}-Z_{1}\right)}-C_{I \mathrm{~m}} e^{\gamma_{m}\left(Z_{3}-Z_{1}\right)}+D_{I \mathrm{~m}} e^{-\gamma_{m}\left(Z_{3}-Z_{1}\right)}=\frac{\sigma_{m}}{\gamma_{m}}
\end{array}
$$$$
\text { at } z=Z_{3}: \quad C_{I I \mathrm{~m}} e^{\gamma_{m} Z_{3}}+D_{I I \mathrm{~m}} e^{-\gamma_{m} Z_{3}}=0
$$

\section{ACKNOWLEDGMENT}

The authors thank the Direction Générale de l'Armement for the financial support.

\section{REFERENCES}

[1] X. Zhang, C. L. Bowman, T. C. O’Connell, et K. S. Haran, "Large electric machines for aircraft electric propulsion », IET Electric Power Applications, vol. 12, $\mathrm{n}^{\circ}$ 6, p. 767-779, 2018.

[2] Y. Terao, W. Kong, H. Ohsaki, H. Oyori, et N. Morioka, «Electromagnetic Design of Superconducting Synchronous Motors for Electric Aircraft Propulsion », IEEE Transactions on Applied Superconductivity, vol. 28, $\mathrm{n}^{\circ}$ 4, p. 1-5, 2018.

[3] K. S. Haran et al., «High power density superconducting rotating machines-development status and technology roadmap », Supercond. Sci. Technol., vol. 30, n ${ }^{\circ}$ 12, p. 123002, 2017.

[4] A. Patel, V. Climente-Alarcon, A. Baskys, B. A. Glowacki, et T. Reis, «Design considerations for fully superconducting synchronous motors aimed at future electric aircraft», IEEE International Conference on Electrical Systems for Aircraft, Railway, Ship Propulsion and Road Vehicles-International Transportation Electrification Conference (ESARS-ITEC), 2018, p. 1-6.

[5] P. J. Masson, K. Ratelle, P. A. Delobel, A. Lipardi, et C. Lorin, « Development of a 3D Sizing Model for All-Superconducting Machines for Turbo-Electric Aircraft Propulsion », IEEE Transactions on Applied Superconductivity, vol. 23, no 3, p. 3600805-3600805, 2013.

[6] K. S. Haran, D. Loder, T. O. Deppen, et L. Zheng, « Actively Shielded High-Field Air-Core Superconducting Machines », IEEE Transactions on Applied Superconductivity, vol. 26, $\mathrm{n}^{\circ}$ 2, p. 98-105, 2016.

[7] E. H. Ailam, D. Netter, J. Lévêque, B. Douine, P. J. Masson, et A Rezzoug, « Design and Testing of a Superconducting Rotating Machine», IEEE Transactions on Applied Superconductivity, vol. 17, no 1, p. $2733,2007$.

[8] P. Masson, J. Lévêque, D. Netter, et A. Rezzoug, « Experimental study of a new kind of superconducting inductor », IEEE Transactions on Applied Superconductivity, vol. 13, no 2, p. 2239 2242, 2003.

[9] C. P. Bean, «Magnetization of Hard Superconductors", Phys. Rev. Lett., vol. 8, no 6, p. 250-253, 1962.

[10] G. P. Mikitik, Y. Mawatari, A. T. S. Wan, et F. Sirois, « Analytical Methods and Formulas for Modeling High Temperature Superconductors », IEEE Transactions on Applied Superconductivity, vol. 23, n 2, p. 8001920-8001920, 2013.

[11] R. Brambilla, F. Grilli, L. Martini, M. Bocchi, et G. Angeli, « A FiniteElement Method Framework for Modeling Rotating Machines With Superconducting Windings », IEEE Transactions on Applied Superconductivity, vol. $28, \mathrm{n}^{\circ} 5$, p. 1-11, 2018.

[12] K. Berger et al., «Benchmark on the 3D Numerical Modeling of a Superconducting Bulk », IEEE Compumag, July 2017.

[13] E. H. Brandt, "Square and Rectangular Thin Superconductors in a Transverse Magnetic Field», Phys. Rev. Lett., vol. 74, $\mathrm{n}^{\circ}$ 15, p. 3025-3028, 1995.
[14] E. H. Brandt, "Thin superconductors in a perpendicular magnetic ac field. II. Circular disk », Phys. Rev. B, vol. 50, nº 6, p. 4034-4050, 1994.

[15] F. Sirois et F. Grilli, «Potential and limits of numerical modelling for supporting the development of HTS devices », Supercond. Sci. Technol., vol. $28, n^{\circ} 4$, p. $043002,2015$.

[16] M. D. Ainslie et H. Fujishiro, «Modelling of bulk superconductor magnetization", Supercond. Sci. Technol., vol. 28, n ${ }^{\circ}$ 5, p. 053002, 2015

[17] K. J. Meessen, B. L. J. Gysen, J. J. H. Paulides, and E. A. Lomonova, "General formulation of fringing fields in 3-D cylindrical structures using Fourier analysis," IEEE Trans. Magn., vol. 48, no. 8, pp. 23072323, Aug. 2012.

[18] Z. Q. Zhu, L. J. Wu, and Z. P. Xia, "An accurate subdomain model for magnetic field computation in slotted surface-mounted permanent magnet machines," IEEE Trans. Magn., vol. 46, no. 4, pp. 11001115,Apr. 2010

[19] J. T. Conway, "Inductance calculations for circular coils of rectangular cross section and parallel axes using Bessel and Struve functions, " IEEE Trans. Magn., vol. 46, no. 1, pp. 75-81, Jan. 2010.

[20] Y. Amara, and G. Barakat, "Analytical modeling of magnetic field in surface mounted permanent-magnet tubular linear machines," IEEE Trans. Magn., vol. 46, no. 11, pp. 3870-3884, Nov. 2010.

[21] O. de la Barrière, S. Hlioui, H. Ben-Ahmed, M. Gabsi, and M. LoBue, "3-D formal resolution of Maxwell equations for the computation of the no-load flux in an axial flux permanent-magnet synchronous machine," IEEE Trans. Magn., vol. 48, no. 1, pp. 128-136, Jan. 2012.

[22] T. Lubin and A. Rezzoug, "Improved 3-D Analytical model for axialflux eddy-current couplings with curvature effects," IEEE Trans. Magn., vol. 53, no. 9, 8002409, Sep. 2017.

[23] G. Malé, T. Lubin, S. Mezani, et J. Lévêque, «2D analytical modeling of a wholly superconducting synchronous reluctance motor», Supercond. Sci. Technol., vol. 24, nº 3, p. 035014, 2011.

[24] W. Rosenheinrich, «Tables Of Some Indefinite Integral Of Bessel Functions Of Integer Order », 2017. Online: http://web.eahjena.de/ rsh/Forschung/Stoer/besint.pdf.

[25] E. Durand, Magnétostatique, MASSON. 1968

[26] P. Mellor, R. Wrobel, et N. Simpson, «AC losses in high frequency electrical machine windings formed from large section conductors », in 2014 IEEE Energy Conversion Congress and Exposition (ECCE), 2014, p. 5563-5570.

[27] J. P. C. Smeets, T. T. Overboom, J. W. Jansen, et E. A. Lomonova, «Three-Dimensional Magnetic Field Modeling for Coupling Calculation Between Air-Cored Rectangular Coils », IEEE Transactions on Magnetics, vol. 47, $\mathrm{n}^{\circ}$ 10, p. 2935-2938, 2011.

[28] G. Malé, T. Lubin, S. Mezani, et J. Lévêque, « Analytical calculation of the flux density distribution in a superconducting reluctance machine with HTS bulks rotor », Mathematics and Computers in Simulation, vol. 90, p. 230-243, 2013.

[29] A. Parviainen, «Design of axial-flux permanent-magnet low-speed machines and performance comparison between radial-flux and axialflux machines », Lappeenranta University of Technology, PHD thesis, 2005

[30] F. N. Werfel et al., « Large-scale HTS bulks for magnetic application », Physica C: Superconductivity, vol. 484, p. 6-11, 2013. 